\title{
Coupled NEGF-PSO Method for Maximizing the Current Ratio of CNTFETs Based on Oxide Thickness Optimization
}

Amin Ghasemi Nejad Raeini ( $\square$ amin_gh1392@yahoo.com )

young research and elite club https://orcid.org/0000-0002-6075-3966

Zoheir Kordrostami

Shiraz University of Technology

Samaneh Hamedi

Shiraz University of Technology

\section{Research Article}

Keywords: Optimization, CNTFET, Current Ratio, Oxide Thickness, PSO algorithm

Posted Date: May 26th, 2021

DOI: https://doi.org/10.21203/rs.3.rs-363572/v1

License: (c) (i) This work is licensed under a Creative Commons Attribution 4.0 International License.

Read Full License

Version of Record: A version of this preprint was published at Journal of Computational Electronics on August 4th, 2021. See the published version at https://doi.org/10.1007/s10825-021-01743-2. 


\title{
Coupled NEGF-PSO Method for Maximizing the Current Ratio of CNTFETs Based on Oxide Thickness Optimization
}

\author{
Amin Ghasemi Nejad Raeini (0000-0002-6075-3966), Zoheir Kordrostami (0000-0001-9958-6977), \\ Samaneh Hamedi (0000-0003-1393-5609) \\ Department of Electrical and Electronic Engineering, Shiraz University of Technology, Shiraz, Iran \\ Corresponding Author: Zoheir Kordrostami \\ amin_gh1392@yahoo.com
}

\begin{abstract}
Carbon nanotube field-effect transistors (CNTFETs) with optimized oxide thicknesses have been proposed. The optimum oxide thickness that provides the maximum current ratio (on/off ratio) has been calculated for each design. The effect of oxide thickness on the on/off ratio has been investigated by changing its value as the independent variable and calculating on state and off state currents. PSO algorithm has been used to find the exact optimum value of the oxide thickness with the objective of having a maximum current ratio that is one of the most important parameters in switching applications. The optimum insulator thickness is calculated for CNTFETs with different chiral vectors, insulator types, channel lengths and source/drain doping levels. For further study of the CNTFETs, performance parameters such as cutoff frequency and transconductance of the devices have also been calculated and studied. The results of the paper show that the CNTFET designers should select the oxide thickness very carefully not only based on reported values in other works. Each design requires its own optimum oxide thickness which provides the maximum on/off current ratio only for that design.
\end{abstract}

Keywords: Optimization, CNTFET, Current Ratio, Oxide Thickness, PSO algorithm

\section{Introduction}

Because of the unique physical and electrical properties of carbon nanotubes (CNTs), they have been paid much attention as electronic material in molecular and nanoscale electronic devices [1,2,3]. The simple and well-defined structure of the single-wall carbon nanotubes encouraged the researchers for conducting both theoretical and experimental studies [4,5]. The electrical and physical properties of CNTs have been extensively studied in the literature [4]. Replacement of the CNTs in channel of MOSFETs, lead to larger current density and modified short channel effects. These are all significant factors that improve the performance of the nano scale FETs [6,7]. Also, larger carrier mobility in CNTs caused higher current density and operation frequency of the CNTFETs.

Performance optimizations of carbon nanotube FETs have been under study for some years [8-10]. As for the gate contact, different insulator types and thicknesses have been used in previous works [5-13]. Changing the insulator thickness, directly changes the charge transport and the band diagram energy in the channel. This shows that the insulator thickness can be used as a critical parameter to modify the device characteristics [6].

Improving the current ratio of conventional CNTFETs and Schottky Barrier CNTFETs (SB-CNTFETs) have been reported in previous studies $[6,12,13]$ in which they have achieved high current ratios by tuning the gate insulator parameters in different temperatures. Finding the optimum insulator thickness is a very important research challenge. Many works in the literature have neglected this and have not used the optimum insulator thicknesses. In [11] and [12] the tuned gate insulator thicknesses have been 
obtained but one cannot claim that these values are the exact optimum values, because an optimization algorithm has not been used. In most of the works on CNTFET designs, the value of the oxide thickness is selected based on the previously reported values. Among the works that discuss the effect of the oxide thickness on the device performance, almost all of them only check some discrete values of the oxide thickness and then conclude what the best thickness is. In this paper, we have used two methods for finding the best oxide thickness:

1- Changing the values of the oxide thickness (discrete values) and calculating their corresponding on/off current ratios. 2- Using PSO (particle swarm optimization) algorithm with the ability of finding exact value of the oxide thickness for which the on/off current ratio is maximum.

In this paper, we propose using PSO for tuning the gate insulator thickness with the goal of achieving maximum on/off current ratio. On/off current ratio is an important parameter of the FETs and characterizes how much is the difference between the off current and the on current. Current ratio is the figure of merit for having better performance, more $\mathrm{I}_{\mathrm{on}}$ and lower leakage current ( $\mathrm{I}_{\mathrm{off}}$ ) for MOS transistors.

The numerical simulation is based on the Poisson equations and self-consistent solution of the twodimensional Green's function $[14,15]$. The optimum oxide thicknesses have been calculated for CNTFETs with different CNT chiralities, oxide materials, channel lengths and source/drain doping concentrations. The objective of the optimization is to obtain the maximum current ratio (on/off ratio). The proposed structures have been designed based on the PSO outputs (optimum gate oxide thickness) which provide the maximum possible on/off ratios. The characteristics of the proposed designs have been compared with each other. Finally, based on the studied designs and the comparisons, the best structures with the ultimate performances have been introduced. The new structures, have optimum current ratios and very high cutoff frequencies and transconductances.

The rest of the paper is organized as follows: a brief review of the PSO algorithm is discussed in section 2. The methodology for the simulation of the CNTFET has been discussed in section 3. In section 4, the results of the PSO algorithm (optimum oxide thicknesses) are presented and the paper is concluded in section 5 .

\section{Particle Swarm Optimization Method}

PSO algorithms (Particle swarm optimization) are inspired from nature population based on metaheuristic algorithms, that originally legalized to Kennedy, Eberhart and Shi [15-18]. These algorithms imitate the behaviour of fishes schooling and birds flocking. The PSO algorithm, try to modify and improve the solutions according to a featured measure with starting from a randomly distributed set of particles [15]. With some interparticle connections and by moving the particles around the search space utilizing a set of simple mathematical explanations, the improvement is performed. These mathematical statements, in basic and simplest form, motion the movement of any particle toward its best experienced status and the swarm's best status so far, along with some random disorder [15]. However, PSO generally utilized and known as a optimization method, also it has roots in computer animation technology and image presentation where Reeves [18] perform and defined a particle system as a set of autonomous individuals working with each other to configure the exterior of a fuzzy object like an explosion or a cloud.

In this paper, two different codes (both written in matlab) linked with each other. One code is dedicated to solving the Poisson and Schrodinger equations self-consistently based on NEGF formalism and the other code is dedicated to PSO optimization algorithm.

Although the search space is one-dimensional, however it is very time consuming for a researcher to find the optimum value of the variable by sweeping it. Usually sweeping of the variable does not lead to a precise result such as what that has been obtained by PSO up to three decimal places. 
As for your comment we added additional explanations about the optimization procedure tosection "Particle Swarm Optimization Method".

Max iteration number for PSO in our paper is 300. Investigating the algorithm shows that after a few iterations the results for the optimum oxide thickness are repeated and the algorithm converges.

We knew the stochastic nature of the PSO algorithm used and experienced what you commented about converging to different solutions in our other works. However, in this paper, the results of the PSO algorithm were repeatable. They value of the oxide thickness was converged very soon to the best case and this result was repeatable.

\section{Simulation Methodology}

Poisson equations and schrödinger have been solved self consistently [7]. For convert the Poisson equations and Schrödinger into matrices, a finite difference scheme has been used. Based on nonequilibrium Green's function (NEGF) method the charge density and the density of states have been calculated. Our proposed model is can calculating the fringing fields, the potential barriers and the band to band tunneling. Also for solving the Poisson equation, Newton-Raphson method has been used.

The electrostatic potential $V(r, z)$ has obtained by solving two-dimensional cylindrical Poisson equation $[18,19]$ :

$$
\frac{\partial^{2} V}{\partial r^{2}}+\frac{1}{r} \frac{\partial V}{\partial r}+\frac{\partial^{2} V}{\partial z^{2}}=-\frac{q}{\varepsilon} \rho(r, z)
$$

Where $\rho(r, z)$ defined the net charge density distribution and $\mathrm{r}$ is CNT diameter. The net charge distribution $\rho\left(r, z_{j}\right)$ is calculated as [20]:

$$
\begin{gathered}
\rho\left(\mathrm{r}=\mathrm{r}_{\mathrm{CNT}}, \mathrm{z}_{\mathrm{j}}\right)=\mathrm{p}\left(\mathrm{z}_{\mathrm{j}}\right)-\mathrm{n}\left(\mathrm{z}_{\mathrm{j}}\right)+\mathrm{N}_{\mathrm{D}}^{+}-\mathrm{N}_{\mathrm{A}^{-}} \\
\rho\left(\mathrm{r} \neq \mathrm{r}_{\mathrm{CNT}}, \mathrm{z}\right)=0
\end{gathered}
$$

Where $\mathrm{r}_{\mathrm{CNT}}$ is the $\mathrm{CNT}$ radius and $\mathrm{N}_{\mathrm{A}}{ }^{-}$and $\mathrm{N}_{\mathrm{D}}{ }^{+}$are the ionized acceptor and donor concentrations, respectively. Inside of the CNT, the Poisson equation reduced to the Laplace equation and because of the existance of charges at the CNT surface, the gate insulator (oxide) regions exclude for the $\mathrm{CNT} /$ oxide interface. Because of the potential density and charge are invariant around the nanotube, the poisson equation is a 2D problem along the tube's length $(\mathrm{z})$ and around the radial direction (r).

Along surface of the nanotube, a matching condition applied in $\varepsilon$ as below $[6,19]$ :

$$
\left.\varepsilon_{\text {ins }} \frac{\partial V}{\partial r}\right|_{R^{+}}-\left.\varepsilon_{0} \frac{\partial V}{\partial r}\right|_{R^{-}}=-\frac{q(p-n)}{2 \pi R \varepsilon_{0}}
$$

Where the gate radius is $\mathrm{R}^{+}$, the nanotube radius is $\mathrm{R}^{-}$and the insulator thickness is tins $=\mathrm{R}^{+}-\mathrm{R}^{-}$.

At the drain side of the CNTFET, Neumann boundary conditions are applied. At the normal component, the electric field at the channel to drain junction is zero. This boundary condition satisfies the charge neutrality at the source and drain junctions. The same zero electric field conditions have been assumed for other boundaries. The Green function of CNT has been calculated from equation5 [6,21]:

$$
G(E)=\left[\left(E+0^{+}\right) I-H-\Sigma_{1}-\Sigma_{2}\right]^{-1}
$$

Where self energies are defined with $\Sigma_{1,2}, \mathrm{E}$ is energy and $\mathrm{H}$ is Hamiltonian matrix. 
Until a self-consistent potential is acquired, the steps of solving the 2D Poisson equations and coupled NEGF must be repeated. By means of the Landauer-Buttiker formula, the drain current for coherent transport under a bias voltage $\mathrm{V}$ can be calculated after the once self-consistency is achieved[21,22,23]:

$$
I=\frac{2 q}{h} \int_{-\infty}^{+\infty} T(E)\left[f\left(E-E_{F S}\right)-f\left(E-E_{F D}\right)\right] d E
$$

Where drain to source transmission defined as $\mathrm{T}(\mathrm{E})$, electron charge is $\mathrm{q}$ and $f\left(E-E_{F S, D}\right)$ are the Fermi distribution functions at the drain and source sides.

experimental result show in integration intervals from a limited lower value than valence band to a limited higher value above the conduction band provides the accurate results. Changes of this value is about $0.2 \mathrm{eV}$ to $0.4 \mathrm{eV}$ [6].

In order to validate the accuracy of our simulation results, we simulated I-V characteristic of a cylindrical transistor geometry with wrapped-around gate and a zigzag CNT with $50 \mathrm{~nm}$ channel length, symmetric $\mathrm{N}$ type source and drain doping and $8 \mathrm{~nm}$ thick $\mathrm{HfO}_{2}$ high- $k$ gate insulator are assumed in Fig. 1. To have a fair comparison, the simulation parameters are chosen based on [22]. As can be seen, our results are in outstanding agreement with the experimental results presented in [22].

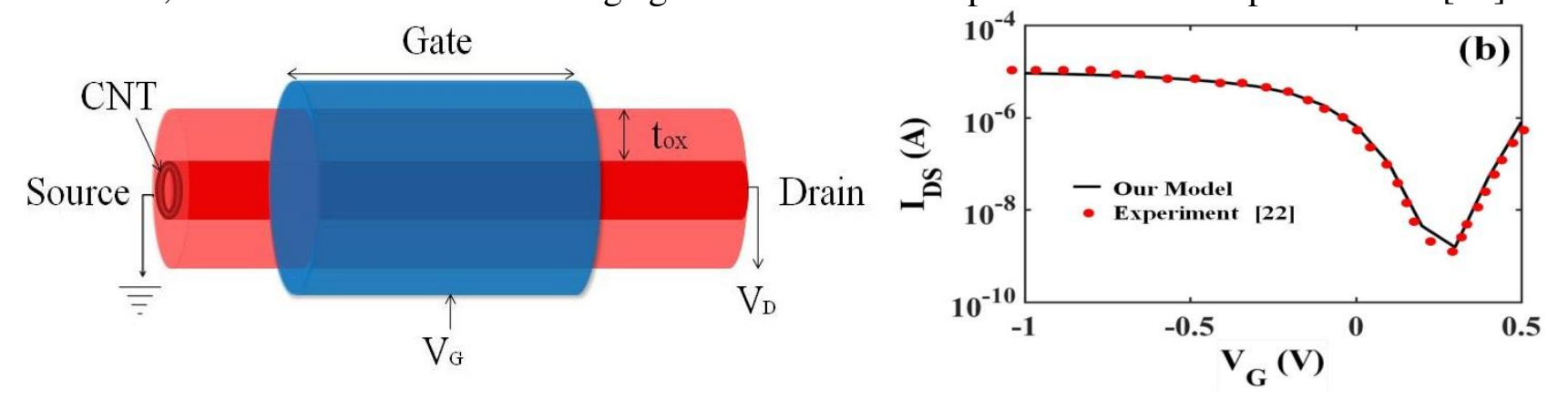

Fig. 1 a) Conventional CNTFET and b) Comparison of the experimental results in [22] and our simulation results.

\subsection{Optimized Oxide thickness for Different Chiralities}

Fig. 2 studies four cases of CNTFETs using CNTs with different chiralities. In Fig. 2 the gate oxide thickness has been changed from $1 \mathrm{~nm}$ to $6 \mathrm{~nm}$ with $1 \mathrm{~nm}$ steps and the thickness for which the on/off current ratio is maximum has been obtained.
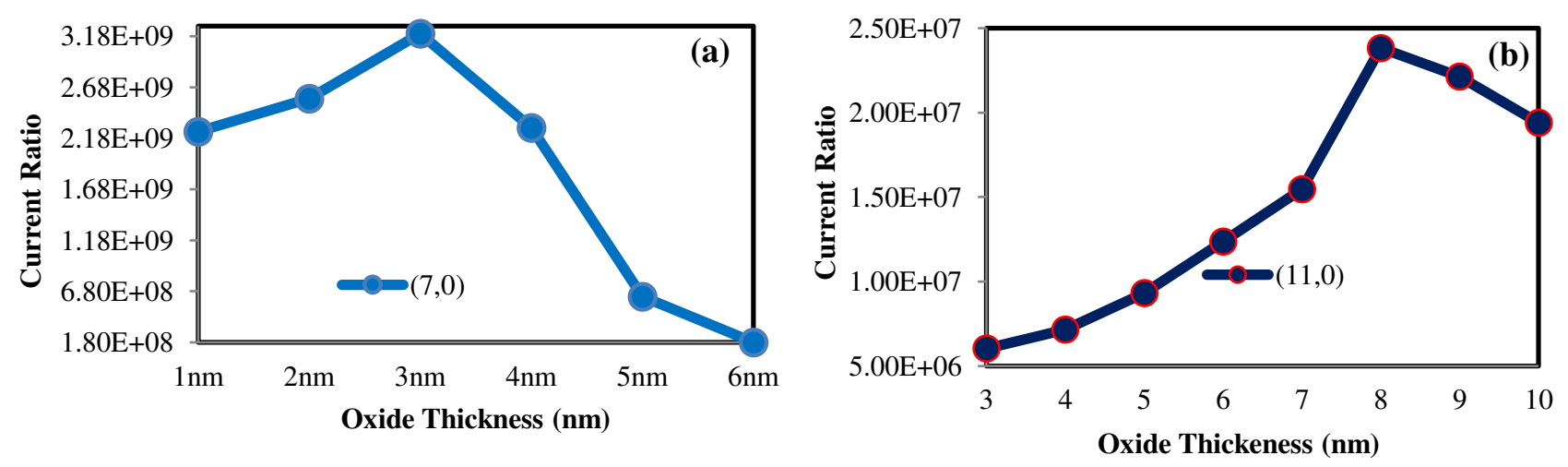

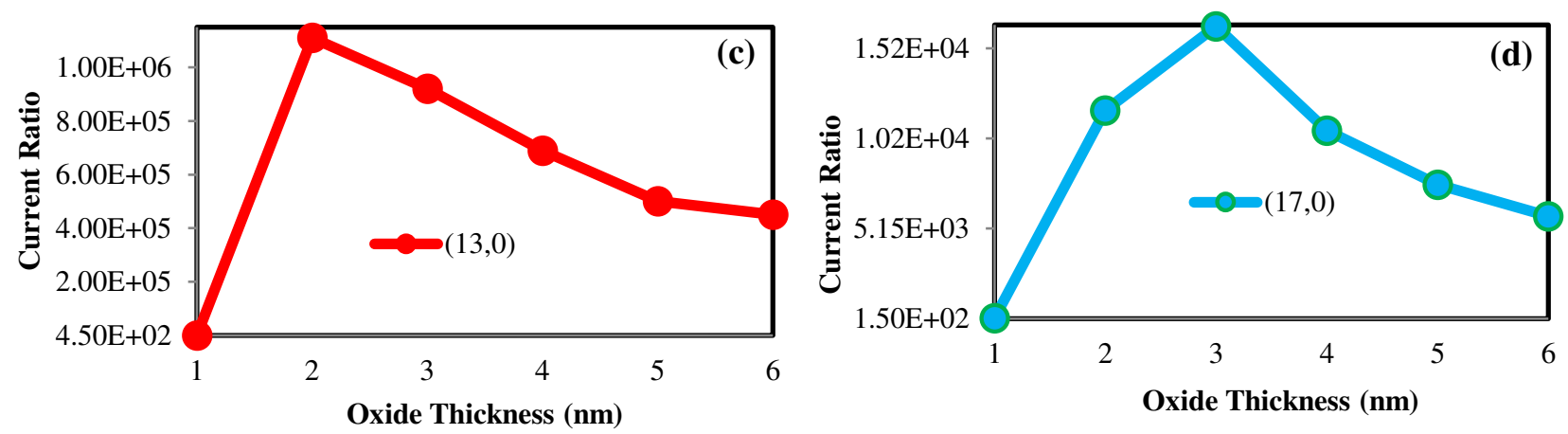

Fig. 2 Current-ratio versus oxide thickness for the same designs of CNTFETs with different chiral vectors for channel length $=20 \mathrm{~nm}$, eps_ins $=16$ and $\mathrm{N}_{\mathrm{sd}}=15 \mathrm{E}+08$.

In Fig. 2, the oxide thickness is changed manually as a discrete parametric variable.

In CNTFETs, two main mechanisms involve in the flow of the electrons from source to drain: the Thermionic Emission (TE) and the Tunneling. The electron transfer can occur over the top of the potential barrier between source and channel via Thermionic Emission (TE) mechanism, or directly from source through the channel via Tunneling.

It should be noted that the general trend for the variations of the current ratio versus the oxide thickness is the same for all chiralities. That is by increasing the oxide thickness the current ratio rises, gets to its maximum value and then decreases. This trend is mainly due to the variations of the off current. For small oxide thicknesses, potential barrier height in the channel is high, so the main component of the leakage current is tunneling mechanism. However, by an increase in the thickness of the oxide, the TE leakage current increases while the tunneling states disappear gradually. These are because of the reduction of the gate field impact on the channel due to the energy band deformation. A further increase in oxide thickness causes the TE transport to become more probable. So the leakage current related to TE mechanism increases and the $\mathrm{I}_{\mathrm{on}} / \mathrm{I}_{\mathrm{off}}$ ratio degrades for thicker oxides. In some intermediate thickness because of intense reduction in tunneling states, an increase in current ratio results, where the leakage current due to tunneling becomes small and the TE leakage current has not dominated yet. This corresponds to emergence of a peak value in on/off current ratio plotted with respect to the oxide thickness. As a conclusion, by increasing the oxide thickness, first the leakage current decreases and the $\mathrm{I}_{\mathrm{on}} / \mathrm{I}_{\mathrm{off}}$ grows. For higher thicknesses, the TE leakage can suppress the effect of reduction in tunneling leakage and thus no further decrease in the off-state current is observed. So, the $\mathrm{I}_{\mathrm{on}} / \mathrm{I}_{\text {off }}$ degradation occurs at higher oxide thicknesses.

In this paper, we aim to find the best oxide thickness which provides the maximum on/off ratio. This can be done by using PSO algorithm. Fig. 3 presents the results of the PSO which shows the optimum gate oxide thicknesses for each chirality. 


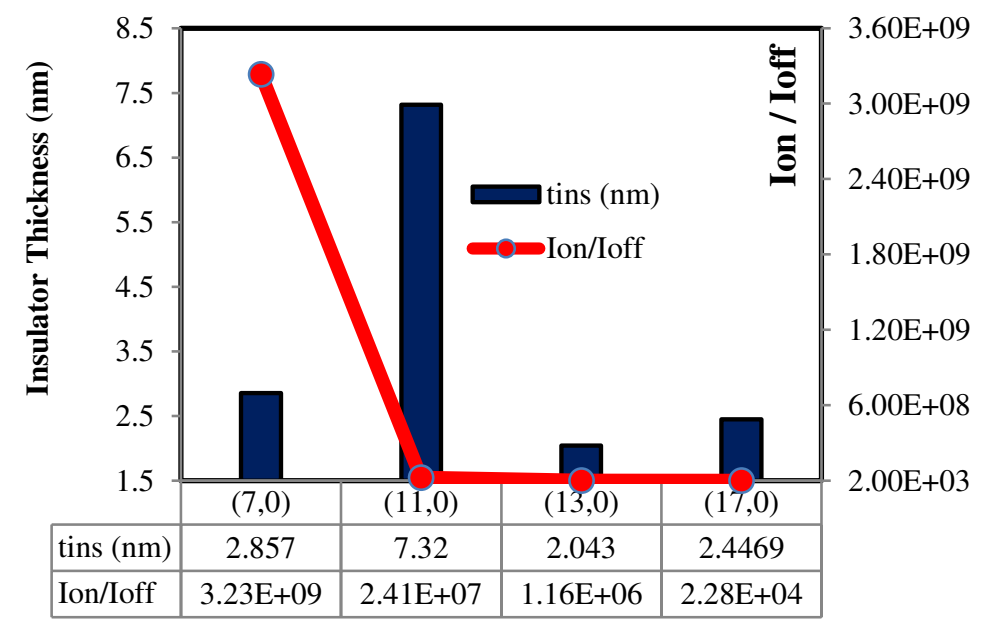

Chiral Vector

Fig. 3 The optimized Current ratio variation of CNTFET for different chiral vector using PSO algorithm for channel length $=20 \mathrm{~nm}$, eps_ins=16, and $\mathrm{N}_{\mathrm{sd}}=15 \mathrm{E}+08$.

It's clear from the results that the lower chiral vector has a higher current ratio. In order to understand the total characteristics of the CNTFET, we have calculated other important transistor parameters such as cutoff frequency and transconductance.

Table 1: Result of PSO algorithm optimization for different parameters.

\begin{tabular}{|l|l|l|l|l|}
\hline Parameter / Type & $(7,0)$ & $(11,0)$ & $(13,0)$ & $(17,0)$ \\
\hline $\mathrm{t}_{\text {ins }}(\mathrm{nm})$ & 2.857 & 7.32 & 2.043 & 2.4469 \\
\hline $\mathrm{I}_{\mathrm{on}} / \mathrm{I}_{\text {off }}$ & $3.23 \mathrm{E}+09$ & $2.41 \mathrm{E}+07$ & $1.16 \mathrm{E}+06$ & $2.28 \mathrm{E}+04$ \\
\hline Cutoff Frequency & $1.11 \mathrm{E}+04$ & $9.50 \mathrm{E}+11$ & $2.11 \mathrm{E}+12$ & $2.11 \mathrm{E}+12$ \\
\hline Transconductance & $3.07 \mathrm{E}-14$ & $2.92 \mathrm{E}-04$ & $6.79 \mathrm{E}-04$ & $1.30 \mathrm{E}-03$ \\
\hline
\end{tabular}

Table 1, shows a complete view of the transistor behavior. From table 1, it can be seen that the $(7,0)$ chiral vector has the highest current ratio, but its cutoff frequency and transconductance are lower than other chiral vectors.

The transconductance is a measure of a transistor sensitivity and shows how sensitive is the device current with respect to the device voltage. It directly affects the gain of the amplifiers and their frequency responses.

The cutoff frequency shows the ultimate frequency in which the transistor is still working correctly. The transistor performance degrades in the frequencies larger than its cutoff frequency.

Also, it is clear from the results that the $(13,0)$ chiral vector provides a higher cutoff frequency and transconductance than other chiral vectors. The $(17,0)$ CNT has a much lower on/off ratio than others. Thus by compromising between the trade-offs, it seems that the $(13,0)$ chirality provides a high cutoff frequency, large enough on/off ratio and a good transconductance. A $(13,0)$ CNT has been used for other optimizations along the paper.

\subsection{Optimized Oxide Thickness for Different Insulator Materials}

By shrinking the transistor size, due to the technology requirements for thinner gate oxides which still block the leakage current, suitable insulator materials such as $\mathrm{ZrO}_{2}, \mathrm{Al}_{2} \mathrm{O}_{3}, \mathrm{HfO}_{2}$, etc with higher dielectric constants have attracted the attentions. The use of materials with high dielectric constants will increase the mobility of electrons and holes in the channel and increases the threshold voltage as well as the current of the transistors. 
The simulation results for the current ratio versus the oxide thickness for different gate insulators have been shown in Fig. 4.
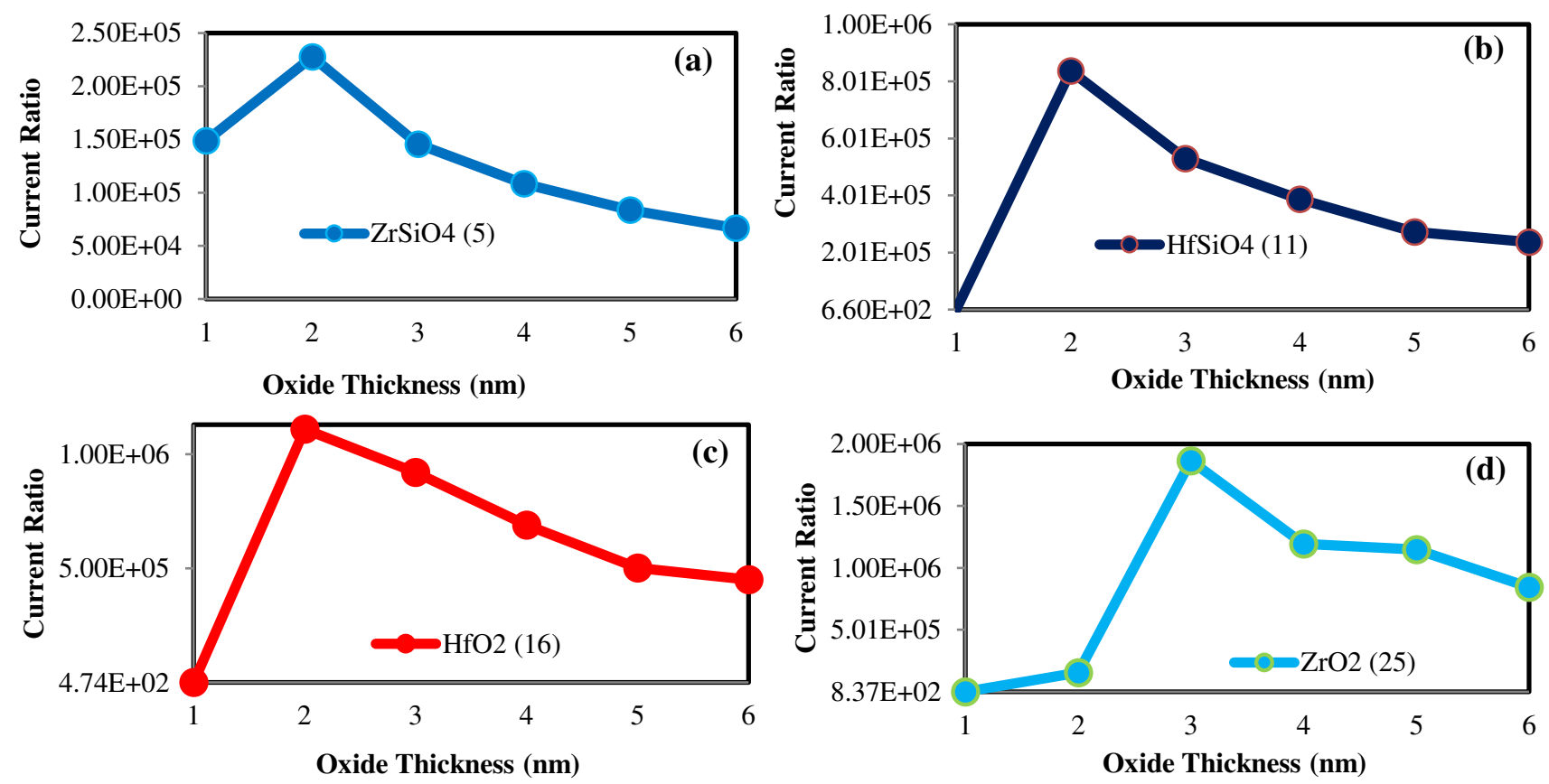

Fig. 4 Current ratio variation of CNTFET versus oxide thickness for different types of oxide for channel length $=20 \mathrm{~nm},(13,0)$ chiral vector and $\mathrm{N}_{\mathrm{sd}}=15 \mathrm{E}+08$.

We have used the PSO algorithm to find the precise value for the oxide thickness for CNTFETs with different insulators with the goal of achieving maximum current ratio and the results of the optimizations are shown in Fig. 5.

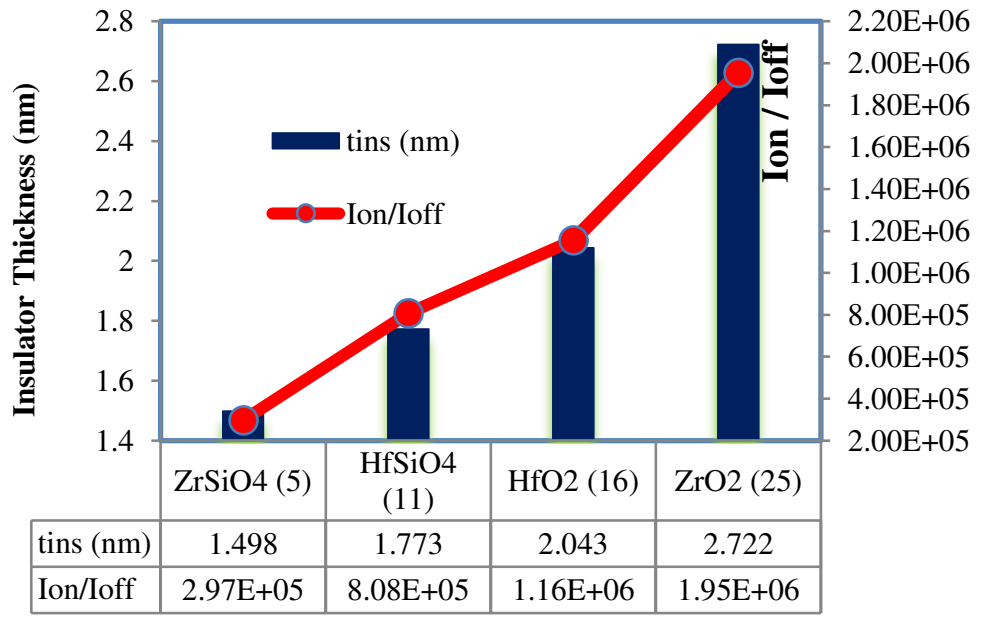

Insulator Type

Fig. 5 Current ratio variation of CNTFET for different types of oxides optimized using PSO algorithm for channel length $=20 \mathrm{~nm},(13,0)$ chiral vector and $\mathrm{N}_{\mathrm{sd}}=15 \mathrm{E}+08$.

As it was expected, the results of Fig. 5 shows that the insulator with a higher dielectric constant has a higher current ratio. The optimum oxide thicknesses and their corresponding on/off ratio have also 
been shown in Fig. 5 for each insulator. In order to better understand the behavior of the CNTFETs, other performance parameters of the optimum designs have been calculated and presented in Table. 2.

Table 2: PSO algorithm results of different parameters at optimized oxide thickness.

\begin{tabular}{|c|c|c|c|c|}
\hline Parameter $/$ Type & $\mathrm{ZrSiO}_{4}(5)$ & $\mathrm{HfSiO}_{4}(11)$ & $\mathrm{HfO}_{2}(16)$ & $\mathrm{ZrO}_{2}(25)$ \\
\hline $\mathrm{t}_{\text {ins }}(\mathrm{nm})$ & 1.498 & 1.773 & 2.043 & 2.722 \\
\hline $\mathrm{I}_{\text {on }} / \mathrm{I}_{\text {off }}$ & $2.97 \mathrm{E}+05$ & $8.08 \mathrm{E}+05$ & $1.16 \mathrm{E}+06$ & $1.95 \mathrm{E}+06$ \\
\hline Cutoff Frequency & $1.55 \mathrm{E}+12$ & $1.94 \mathrm{E}+12$ & $2.11 \mathrm{E}+12$ & $2.31 \mathrm{E}+12$ \\
\hline Transconductance & $3.21 \mathrm{E}-04$ & $5.48 \mathrm{E}-04$ & $6.79 \mathrm{E}-04$ & $8.41 \mathrm{E}-04$ \\
\hline
\end{tabular}

From the data in Table. 2, it can be understood that the $\mathrm{ZrO}_{2}$ insulator has a higher current ratio, cutoff frequency and transconductance at its optimum thickness value. Thus, it can be concluded that the $\mathrm{ZrO}_{2}$ insulator with a thickness of $2.722 \mathrm{~nm}$ is the best case among the studied designs.

\subsection{Optimized Oxide Thickness for Different Gate Lengths}

In this section, the simulation results for the calculation of the current ratio versus the oxide thickness for different channel lengths are shown in Fig. 6.
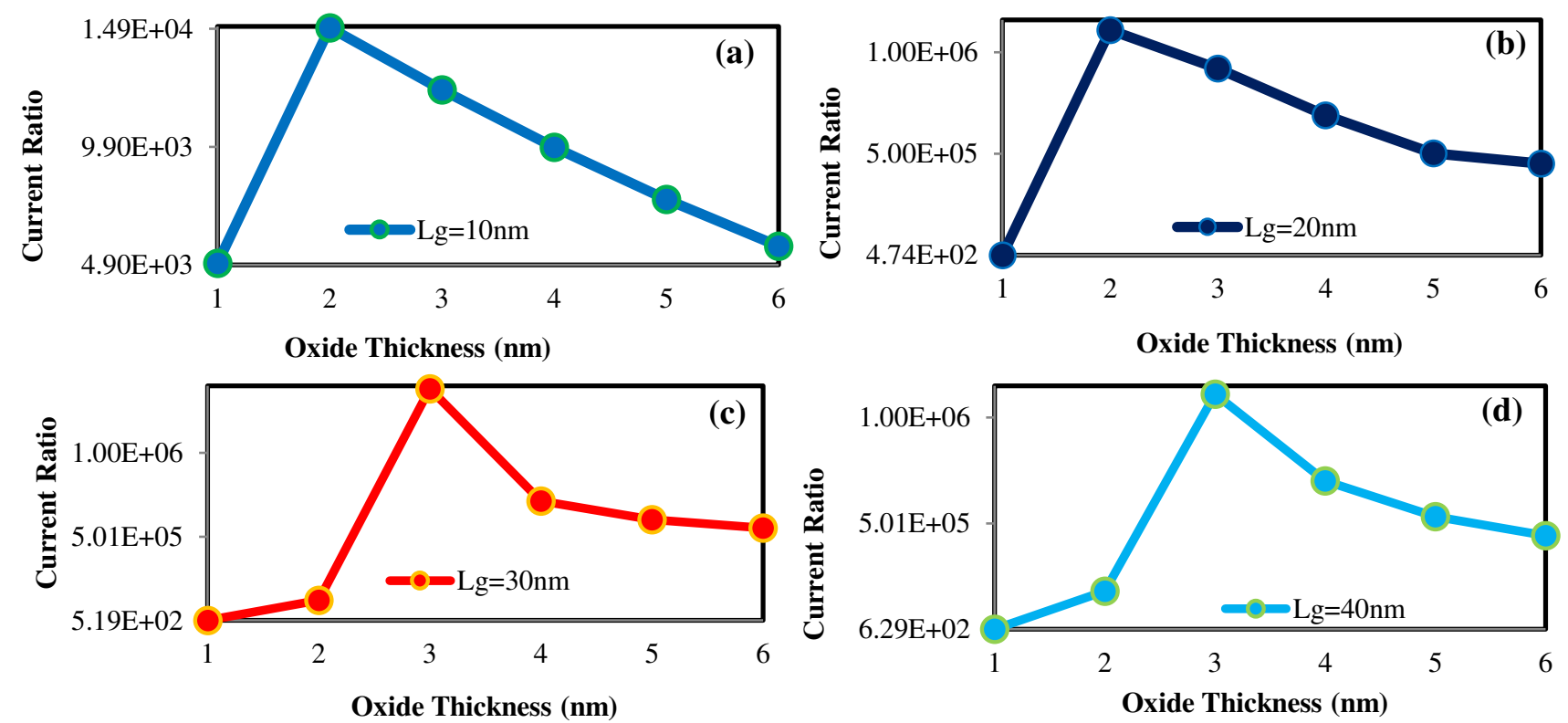

Fig. 6 Current ratio variation of CNTFET versus different oxide thicknesses for different channel lengths for $(13,0)$ chiral vector, eps_ins=16, and $\mathrm{N}_{\mathrm{sd}}=15 \mathrm{E}+08$.

As can be seen in fig. 6, lower channel length leads to a lower current ratio. That is because of the increase in the off current. In order to calculate the precise optimum oxide thickness for each channel length, the PSO algorithm has been used and its optimization results have been shown in Fig. 7. 


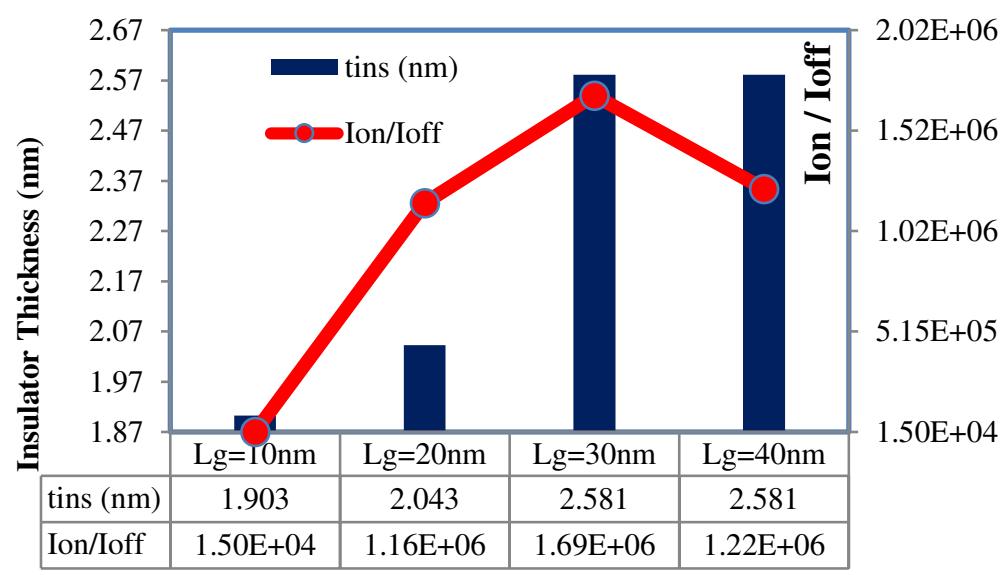

Channel Length

Fig. 7 The optimized Current ratio variation of CNTFET for different channel lengths using PSO algorithm for $(13,0)$ chiral vector, eps_ins $=16$, and $\mathrm{N}_{\mathrm{sd}}=15 \mathrm{E}+08$.

Table 3: PSO algorithm results of different parameters at optimized oxide thickness.

\begin{tabular}{|l|c|c|c|c|}
\hline Parameter $/$ Type & $\mathrm{L}_{\mathrm{g}}=10 \mathrm{~nm}$ & $\mathrm{~L}_{\mathrm{g}}=20 \mathrm{~nm}$ & $\mathrm{~L}_{\mathrm{g}}=30 \mathrm{~nm}$ & $\mathrm{~L}_{\mathrm{g}}=40 \mathrm{~nm}$ \\
\hline $\mathrm{t}_{\text {ins }}(\mathrm{nm})$ & 1.903 & 2.043 & 2.581 & 2.581 \\
\hline $\mathrm{I}_{\text {on }} / \mathrm{I}_{\text {off }}$ & $1.50 \mathrm{E}+04$ & $1.16 \mathrm{E}+06$ & $1.69 \mathrm{E}+06$ & $1.22 \mathrm{E}+06$ \\
\hline Cutoff Frequency & $3.53 \mathrm{E}+12$ & $2.11 \mathrm{E}+12$ & $1.60 \mathrm{E}+12$ & $1.27 \mathrm{E}+12$ \\
\hline Transconductance & $6.9 \mathrm{E}-04$ & $6.79 \mathrm{E}-04$ & $6.75 \mathrm{E}-04$ & $6.75 \mathrm{E}-04$ \\
\hline
\end{tabular}

Other important parameters are calculated and presented in Table 3. It can be seen in Table 3 that the $30 \mathrm{~nm}$ channel length has a higher current ratio than other structures at optimum oxide thickness. But at this optimum oxide thickness, the $30 \mathrm{~nm}$ channel length has a slightly lower cutoff frequency than other lengths like $10 \mathrm{~nm}$ and $20 \mathrm{~nm}$. Also, at the $30 \mathrm{~nm}$ channel length, the transconductance is near to other channel lengths.

\subsection{Optimized Oxide Thickness for Different Doping Concentrations}

In this section, the oxide thickness has been optimized for different doping concentrations. The simulation results of the current ratio versus oxide thickness for different doping concentrations are shown in Fig. 8.
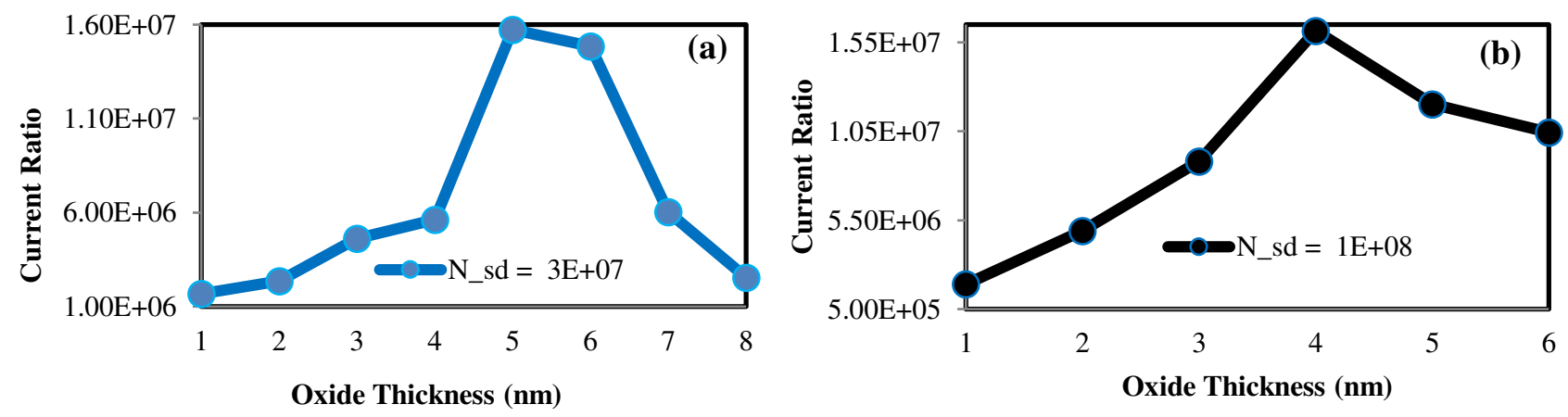

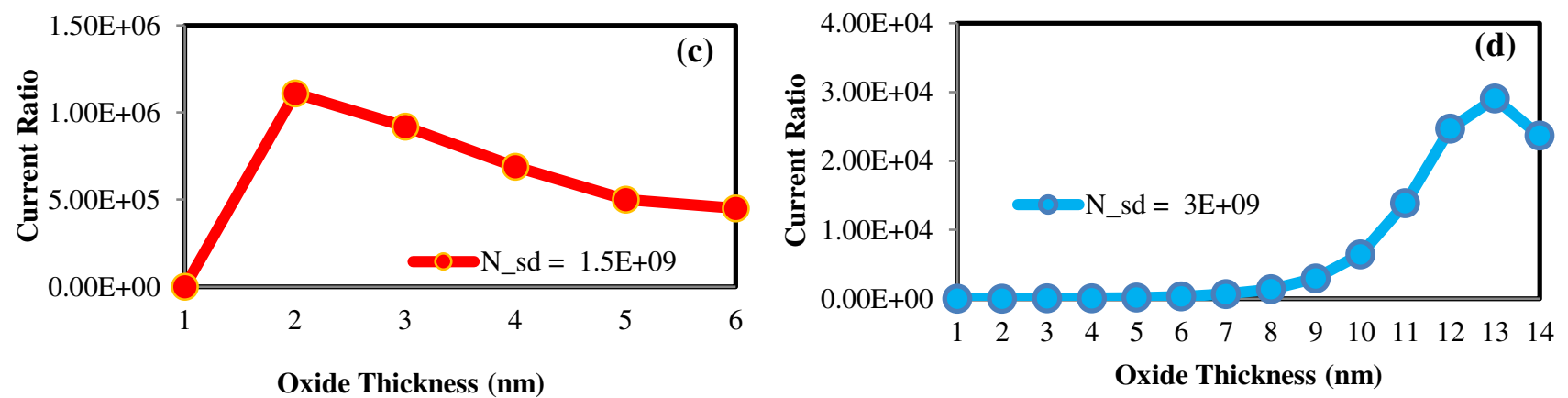

Fig. 8 Current ratio variation of CNTFET versus different oxide thickness for different doping levelsfor a $(13,0)$ chiral vector, eps_ins $=16$, and channel length $=20 \mathrm{~nm}$.

In figure 8 , for every doping concentration, the oxide thickness has been varied and the on/off ratio has been calculated. However, in order to find the exact value of the optimum oxide thickness which corresponds to the maximum on/off ratio, the PSO algorithm has been used. The outputs of the PSO have been shown in Fig. 9.

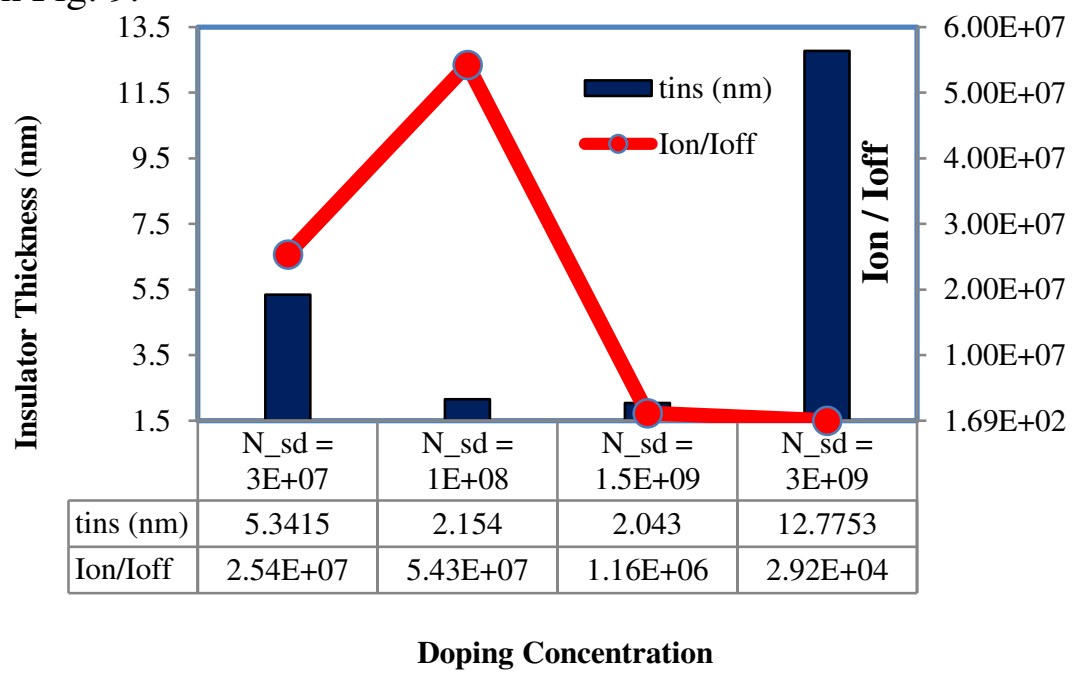

Fig. 9 The optimized current ratio of conventional CNTFET for different doping concentrations using the PSO algorithm at $V_{D}=0.5 \mathrm{~V}$.

It is clear from Fig. 9 that the highest current ratio has been obtained for $\mathrm{N}_{\mathrm{sd}}=1 \mathrm{E}+08$ source and drain doping concentration. Other important parameters for CNTFET with $\mathrm{N}_{\mathrm{sd}}=1 \mathrm{E}+08$ have been calculated and shown in Table 4.

Table 4: PSO algorithm results of different parameters at optimized oxide thickness.

\begin{tabular}{|l|l|l|l|l|}
\hline Parameter / Type & $\mathrm{N}_{-s d}=3 \mathrm{E}+07$ & $\mathrm{~N}_{-} \mathrm{dd}=1 \mathrm{E}+08$ & $\mathrm{~N}_{-} \mathrm{sd}=1.5 \mathrm{E}+09$ & $\begin{array}{l}\mathrm{N}_{-s d} \\
3 \mathrm{E}+09\end{array}$ \\
\hline $\mathrm{t}_{\text {ins }}(\mathrm{nm})$ & 3.098 & 2.154 & 2.043 & 12.7753 \\
\hline $\mathrm{I}_{\text {on }} / \mathrm{I}_{\text {off }}$ & $1.08 \mathrm{E}+07$ & $5.43 \mathrm{E}+07$ & $1.16 \mathrm{E}+06$ & $2.92 \mathrm{E}+04$ \\
\hline Cutoff Frequency & $2.73 \mathrm{E}+12$ & $3.45 \mathrm{E}+12$ & $2.11 \mathrm{E}+12$ & $2.41 \mathrm{E}+12$ \\
\hline Transconductance & $8.32 \mathrm{E}-05$ & $8.25 \mathrm{E}-04$ & $6.79 \mathrm{E}-04$ & $2.60 \mathrm{E}-03$ \\
\hline
\end{tabular}

The simulation results show that the optimum doping level with the optimized insulator thickness has improved the device performance. It can be seen in Table. 4, that the source and drain doping 
concentration $\mathrm{N}_{\mathrm{sd}}=1 \mathrm{E}+08$ provides a higher current ratio, cutoff frequency, and transconductance than other doping levels at the optimum oxide thickness. The optimized high performance CNTFETs designed based on the obtained results have been proposed in next section.

\section{Proposed Optimized CNTFETs}

In this section, based on the optimum values and the obtained results in previous sections, two CNTFET structures with different chiral vectors have been proposed.

The design parameters and the DC and AC characteristics of the proposed CNTFET with a $(7,0)$ CNT have been shown in Table 5. As can be seen, for a $30 \mathrm{~nm}$ long CNT and a $3.114 \mathrm{~nm}$ thick oxide with a dielectric constant of 25 , the on/off ratio has reached $5.73 \mathrm{E}+09$ and a $4.03 \mathrm{E}+11 \mathrm{~Hz}$ cutoff frequency has been obtained. For further understanding of the behavior of the device, other parameters such as PDP and delay have been also shown in Table 5.

Table 5: PSO algorithm results of first proposed structure with optimized oxide thickness with a $(7,0)$ chiral vector.

\begin{tabular}{|c|c|c|c|c|c|}
\hline $\begin{array}{l}\text { Parameter } \\
\text { /Type } \\
\end{array}$ & Ion/Ioff & \begin{tabular}{|l} 
Cutoff \\
Frequency
\end{tabular} & Transconductance & PDP & Delay \\
\hline$N_{-s d}=1 E+08$ & \multirow{5}{*}{$5.73 \mathrm{E}+09$} & \multirow{5}{*}{$4.03 \mathrm{E}+11$} & \multirow{5}{*}{$4.59 \mathrm{E}-08$} & \multirow{5}{*}{$4.94 \mathrm{E}+20$} & \multirow{5}{*}{$3.96 \mathrm{E}+10$} \\
\hline $\mathrm{L}_{\mathrm{g}}=30 \mathrm{~nm}$ & & & & & \\
\hline eps_ins $=25$ & & & & & \\
\hline Chiral Vector $=(7,0)$ & & & & & \\
\hline $\mathrm{t}_{\mathrm{ins}}=3.114 \mathrm{~nm}$ & & & & & \\
\hline
\end{tabular}

The same procedure has been used to get to a high performance utilizing a $(13,0)$ CNTFET. The design parameters of the proposed CNTFET has been shown in Table 6.

Table 6: PSO algorithm results of second proposed structure with optimized oxide thickness at $(13,0)$ chiral vector.

\begin{tabular}{|c|c|c|c|c|c|}
\hline $\begin{array}{l}\text { Parameter } \\
\text { /Type } \\
\end{array}$ & $\mathrm{I}_{\mathrm{on}} / \mathrm{I}_{\mathrm{off}}$ & $\begin{array}{c}\text { Cutoff } \\
\text { Frequency }\end{array}$ & Transconductance & $\underline{\text { PDP }}$ & $\underline{\text { Delay }}$ \\
\hline $\mathrm{N}_{\text {sd }}=1 \mathrm{E}+08$ & \multirow{5}{*}{$7.21 \mathrm{E}+07$} & \multirow{5}{*}{$2.03 \mathrm{E}+12$} & \multirow{5}{*}{$1.50 \mathrm{E}-03$} & \multirow{5}{*}{$6.33 \mathrm{E}-19$} & \multirow{5}{*}{$1.69 \mathrm{E}-13$} \\
\hline $\mathrm{L}_{\mathrm{g}}=30 \mathrm{~nm}$ & & & & & \\
\hline eps_ins $=25$ & & & & & \\
\hline Chiral Vector $=(13,0)$ & & & & & \\
\hline $\mathrm{t}_{\mathrm{ins}}=11.4 \mathrm{~nm}$ & & & & & \\
\hline
\end{tabular}

It's notable from the comparison between table. 5 and table. 6 , that the $(7,0)$ chiral vector has larger current ratio and delay and lower cutoff frequency and transconductance. This is due to the different bandgap of the CNTs used in these structures.

The results of the paper show that the CNTFET designers should select the oxide thickness very carefully not only based on the previously reported values in other works. Each design requires its own optimum oxide thickness that provides the maximum on/off current ratio, which is one of the electrical figures of merit (FOM) for the optimized structure. The current ratio is an important FOM for digital applications while transconductance (gm) plays an important role in analogue applications of a device where the amplification is necessary. Therefore, it is not necessary or even practical to optimize a device for both applications. 


\section{Conclusion}

This work showed that most of the works on CNTFET's has not paid attention to the importance of the exact optimum value of the oxide thickness. In this paper, the optimum oxide thicknesses were calculated based on the PSO algorithm with the objective of maximizing the on/off current ratio of the CNTFETs. The results showed that due to change in the contributions of the tunnelling and thermionic emission leakage currents with change of the oxide thickness, every CNTFET has a peak value of on/off ratio in an intermediate oxide thickness. The important result is that by changing the chirality, the channel length, the doping concentration and the insulator type, the optimum value for the oxide thickness changes. By calculation of the transconductance and the cutoff frequency as well as the on/off ratio, the whole device performance was studied. It was shown that the CNTFET's characteristics have high dependence on the oxide thickness and it considerably affects its ac and dc performance.

Funding statement: Funding information is not applicable.

Conflict of interest: The authors listed in this paper, certify that they have no affiliations with any financial interest involvement in any organization, or nonfinancial interest in the subject matter or any discussed in this manuscript.

\section{Author contributions:}

1: Study concept and design; data acquisition; Statistical analysis; Analysis and interpretation of data. 2: Study supervision; Administrative, technical, and material support; Critical revision of the manuscript for important intellectual content.

3: Revision of the manuscript for important intellectual content

Availability of data and material: All data that support the figures and tables of this study are available on request.

Compliance with ethical standards: This paper complied by the university human research ethics committee and all methods used in other studies involving human participants were in accordance with national research committee, the ethical standards of the institutional, its later correction or comparable ethical standards and with the 1964 Helsinki declaration.

Consent to participate: Informed agreement was obtained from authors included in this study.

Consent for publication: Informed agreement was obtained from authors included in this study.

Acknowledgments: This study has no Acknowledgments.

\section{References}

1. Zhi Chen, "Nanotubes for Nanoelectronics. Encyclopedia of Nanoscience and Nanotechnology," 7-920 (2004).

2. R. E. Smalley, Available: http://www.ruf.rice.edu/ smalleyg/image gallery. htm (2002).

3. Vahideh Khademhosseini, Daryoosh Dideban, MohammadTaghi Ahmadi and Razali Ismail, "Impact of Chiral Indices on the Performance of Single Electron Transistor Utilizing Carbon Nanotube Island" ECS Journal of Solid State Science and Technology, 8 (3) 26-29 (2019). 
4. R Saito, M. S. Dresselhaus, and G. Dresselhaus, Physical Properties of Carbon Nanotubes. World Scientific (1998).

5. K. Tanaka, T. Yamabe, and K. Fukui, The Science and Technology of Carbon Nanotubes. Elsevier (1999).

6. Amin Ghasemi Nejad Raeini, Zoheir Kordrostami, "Modified Schottky Barrier CNTFET with lightly doped drain," IET Micro \& Nano Letters, 13, 1-5 (2018).

7. Kumar Prasannajit Pradhan, Kumar Prasannajit Sahu, "Benefits of asymmetric underlap dual-k spacer hybrid fin field-effect transistor over bulk fin field-effect transistor," IET Circuits, Devices \& Systems, 10, 441-447 (2016).

8. Wei Wang, Huan Wang, "Performance analysis of an ultralow power circuit using single halo CNTFETs," IOP Semiconductor Science and Technology, 30, 1-9 (2015).

9. Zoheir Kordrostami, M. Hossein Sheikhi, and Abbas Zarifkar, "Influence of Channel and Underlap Engineering on the High-Frequency and Switching Performance of CNTFETs," IEEE Transactions on Nanotechnology, 11, 526-533 (2012).

10. Hadi owlia, Parviz Keshavarzi, "Locally Defect-Engineered Graphene Nanoribbon Field-Effect Transistor," IEEE Transactions on Electron Devices, 63, 3769 - 3775 (2016).

11. Shaahin G. Shirazi, Sattar Mirzakuchaki, "High on/off current ratio in ballistic CNTFETs based on tuning the gate insulator parameters for different ambient temperatures," Appl Phys A, 113, 447-457 (2013).

12. Amin Ghasemi Nejad Raeini, Zoheir Kordrostami, Mojtaba Javaheri, "High On/Off Current Ratio in SBCNTFET Based on Tuning the Gate Insulator Parameters for Different Ambient Temperatures," presented at the IEEE-NEMS, china, 630-634 (2015).

13. Amin Ghasemi Nejad Raeini, Zoheir Kordrostami, "Asymmetric lightly doped Schottky barrier CNTFET," IET Micro \& Nano Letters, 11, 1-5 (2016).

14. Amin Ghasemi Nejad Raeini, Mehran Abdali, "Performance Optimization of Conventional CNTFETs Based on Asymmetric Lightly Doped Source and Drain Regions," ECS Journal of solid state Science and technology, 5, 23-26 (2016).

15. Kaveh, "Advances in Metaheuristic Algorithms for Optimal Design of Structures", Springer International Publishing (2017).

16. Kennedy J, Eberhart R, "Particle swarm optimization,” Proc IEEE Int Conf Neural, 1942 (1995).

17. Shi Y, Eberhart R, "A modified particle swarm optimizer" presented at 1998 the IEEE World Congress on computational intelligence, 69-73 (1998).

18. Reeves WT, "Particle systems a technique for modeling a class of fuzzy objects," ACM Trans Graph, 2, 91$108(1983)$.

19. Geunho Cho, Yong-Bin Kim, Fabrizio Lombardi, "Assessment of CNTFET Based Circuit Performance and Robustness to PVT Variations, "presented at the 2009 IEEE International Midwest Symposium on Circuits and Systems, 1106-1109 (2009). 
20. Sudipta Bardhan, Manodipan Sahoo, Hafizur Rahaman, "Boltzmann transport equation-based semiclassical drain current model for bilayer GFET including scattering effects," IET Circuits, Devices \& Systems, 13, 456-464 (2019).

21. Ajay Kumar Singh, B. Naresh Kumar, G. Ramana Murthy, C.M.R. Prabu, "A comprehensive analytical study of electrical properties of carbon nanotube field-effect transistor for future nanotechnology," Int J Numer Model, 31, 1-9 (2017).

22. Ali. Javey, Jing. Guo, etal, "Self-Aligned Ballistic Molecular Transistors and Electrically Parallel Nanotube Arrays," NANO LETTERS, 4, 1319-1322 (2004).

23. Hosseini, V. K., Dideban, D., Ahmadi, M. T., Ismail, R. and Heidari, H, "Single Electron Transistor Scheme Based on Multiple Quantum Dot Islands: Carbon Nanotube and Fullerene," ECS Journal of Solid State Science and Technology, 7(10), 145-152 (2018). 